\title{
Dependency of magnetic material load and feed excitation on the magnetic field distribution of two linearly aligned solenoids for near field communication
}

\author{
Yoshihiro Nakamura, Keizo Cho ${ }^{\text {a) }}$, and Hiroaki Nakabayashi \\ Graduate School of Engineering, Chiba Institute of Technology, \\ 2-17-1 Tsudanuma, Narashino-shi, Chiba 275-0016, Japan \\ a)cho.keizo@it-chiba.ac.jp
}

Abstract: The miniaturization of conventional near field communication (NFC) antennas is required to improve the flexibility of placement of the constituent electrical and electronic components. A small-sized magnetic material loaded solenoid has been proposed. However, the transmission efficiency of this design decreases as the size of the antenna decreases.

This study focuses on a linearly aligned, two-solenoid array and numerically investigates the dependency of the magnetic field distribution radiated from the solenoid array in relation to the excitation direction. The influences of the magnetic material loaded into the solenoids on the magnetic field distribution are also analyzed. Based on the results, the out-of-phase excitation can yield larger magnetic field strengths near the center of the solenoid array compared to the in-phase excitation because the total magnetic field produced by the two solenoids in the former case is the summation of the magnetic fields of the constituent fields generated within the tested area. When the observation point is far from the solenoid, the in-phase excitation can generate a larger magnetic field strength than the out-of-phase excitation because the magnetic field parallel to the solenoid becomes dominant. In addition, it is found that the excitation of the linearly aligned, two-solenoid array suitable for NFC depends on the permeability of the magnetic material and the target distance between the solenoid array and the receiving antenna.

Keywords: solenoid, transmission efficiency

Classification: Antennas and Propagation

\section{References}

[1] M. Takahashi, Antenna Design for RFID Tags, Corona Publishing Co., Ltd., Tokyo, Japan, 2012. 
[3] ANSYS, “ANSYS HFSS,” https://www.ansys.com/Products/Electronics/ ANSYS-HFSS, accessed May 31, 2019.

\section{Introduction}

Because of the widespread advent of contactless integrated circuits (ICs) in communications in recent years, such as FeliCa, near field communication (NFC) antennas have been extensively installed in mobile terminals. Given that NFC operates based on the use of a magnetic field, planar-loop and spiral antennas have been extensively used in view of their capacities to be mounted on card-type terminals [1].

Meanwhile, owing to the continuous demands for higher mobile terminal performances, the miniaturization of the conventional NFC antennas is required to improve the flexibility of the placement of the constituent electrical and electronic parts.

Accordingly, a solenoid loaded with a magnetic material with an approximate diameter of $1 \mathrm{~mm} \phi$ has been previously proposed [2]. However, the shortcoming of the design is attributed to the fact that the transmission efficiency decreases as the size of the antenna decreases. To overcome this shortcoming, we investigate herein an array configuration comprising small-sized solenoids because separate configurations make the antenna and the placement of the electrical/electronic components more flexible compared to the use of large-sized solenoids.

Herein, we focused on a linearly aligned, two-solenoid array. There are two ways to feed the two solenoids. The first uses in-phase excitation and the other outof-phase excitation. In the in-phase excitation case, the directions of the magnetic fields penetrating the two solenoids are the same. In this respect, it phenomenologically appears that the length of the solenoid is enlarged. Thus, it is expected that the strength of the radiated magnetic field increases. In the latter case, the magnetic fields radiated from the two solenoids are added in between the solenoids and in the direction perpendicular to the array direction. Thus, the magnetic field strength is expected to increase along these directions. Correspondingly, it is critical to assess which excitation is suitable for the NFC.

In this study, we investigated numerically the dependency of the magnetic field distribution radiated from the linearly aligned, two-solenoid array in relation on the excitation direction. The influences of the magnetic material loaded into the solenoids on the magnetic field distribution were also analyzed. The commercial full-wave simulator based on a finite-element method, ANSYS HFSS [3], is used for simulations and analyses.

\section{Solenoid structure}

Fig. 1(a) shows the solenoid structure used for simulations and analyses. The crosssection of the solenoid is rectangular with a height of $0.785 \mathrm{~mm}$ and a width of $0.930 \mathrm{~mm}$, and the solenoid is configured with the use of six copper strips that are arranged in parallel with $0.1 \mathrm{~mm}$ spacings between the centers of the adjacent 
copper strips. The six strips are wound simultaneously 20 times, as shown in Fig. 1(a). These strips are connected to copper plates at both ends. The length of the solenoid is $12.55 \mathrm{~mm}$ and the widths and thicknesses of all the copper strips are $50 \mu \mathrm{m}$, respectively. Two types of solenoids are considered in this study. One is an air-core solenoid (ACS), and the other is a magnetic core solenoid (MCS). The structures of both solenoids are the same. The shape of the magnetic material is a rectangular solid whose height, width, and length, are $0.685 \mathrm{~mm}, 0.830 \mathrm{~mm}$, and $12.55 \mathrm{~mm}$, respectively. The relative permeability of the magnetic material is 100 . Other structural parameters are shown in Fig. 1(a). The frequency at which the coils were tested was $13.56 \mathrm{MHz}$.

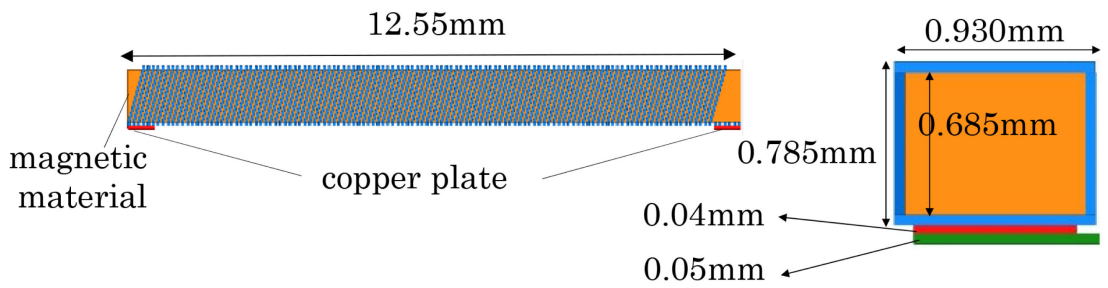

(a) Structure of magnetic material loaded solenoid

Observation position of magnetic field strength distance characteristic

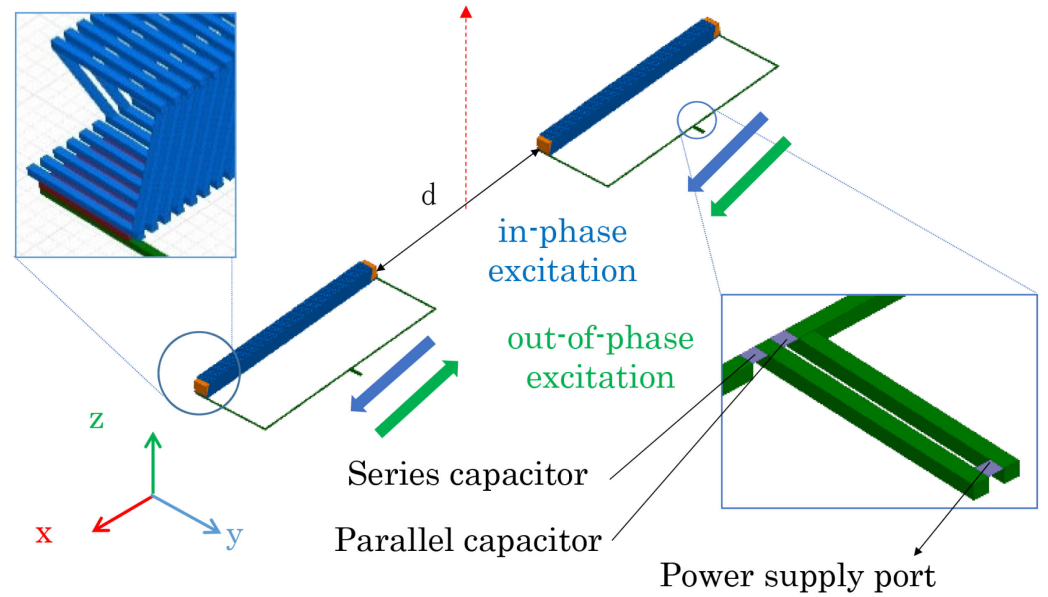

(b) Structure of linearly arranged solenoid

Fig. 1. Solenoid array structure.

\section{Magnetic field distribution in relation to the feed and loading conditions}

Fig. 2 shows the magnetic field strength along the $\mathrm{z}$ axis located at the center of the two-solenoid array, as indicated by the red-dashed line shown in Fig. 1(b). In this case, $\mathrm{z}=0$ coincides with the bottom part of the solenoid. The ACS and MCS arrays are considered and the in-phase and out-of-phase excitations are investigated in each solenoid array configuration. Fig. 3 shows the magnetic field distributions in the zx plane for each of the four configurations.

As shown in Fig. 2, the magnetic field strengths of the in-phase excitation are larger than those of the out-of-phase excitation in both the ACS and MCS array configurations when $\mathrm{z}$ is less than several millimeters. This is because the direction 
of the magnetic field of the solenoid in the right side is the same as that of the left solenoid near the cross-sectional surface of the solenoids, and especially that shown in Area 1 in Fig. 3. Therefore, these magnetic fields are added. However, given that the magnetic field flows in a loop form at both edges of each solenoid, the magnetic fields of the left and right ACSs are cancelled out in the in-phase excitation case in Area 2, as shown in Fig. 3(a). Conversely, the magnetic fields of the out-of-phase excitation are added in Area 2, as shown in Fig. 3(b). Thus, the magnetic field strength of the out-of-phase excitation is larger than that of the in-phase excitation in this area. When $\mathrm{z}$ increases further, the magnetic fields radiated from the left and right ACSs are added, while the magnetic fields radiated from the solenoids are cancelled out in the out-of-phase excitation case given that the direction of the

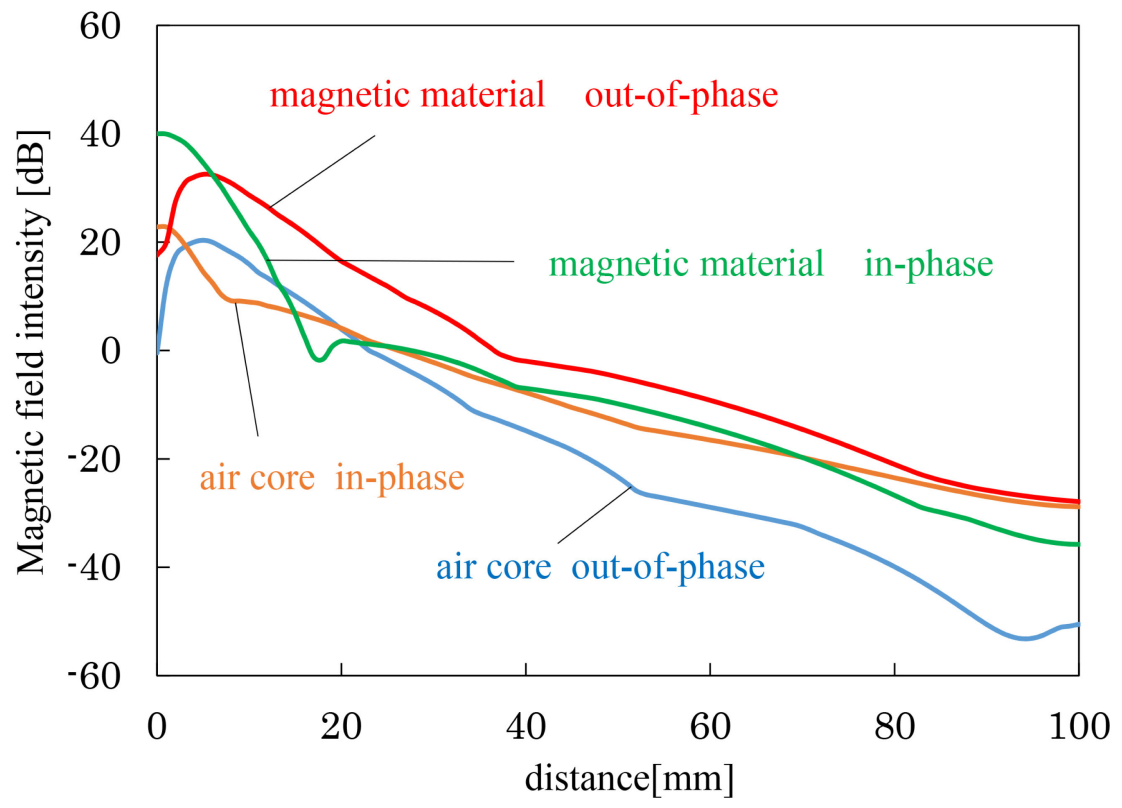

Fig. 2. Variations of magnetic field strength as a function distance.

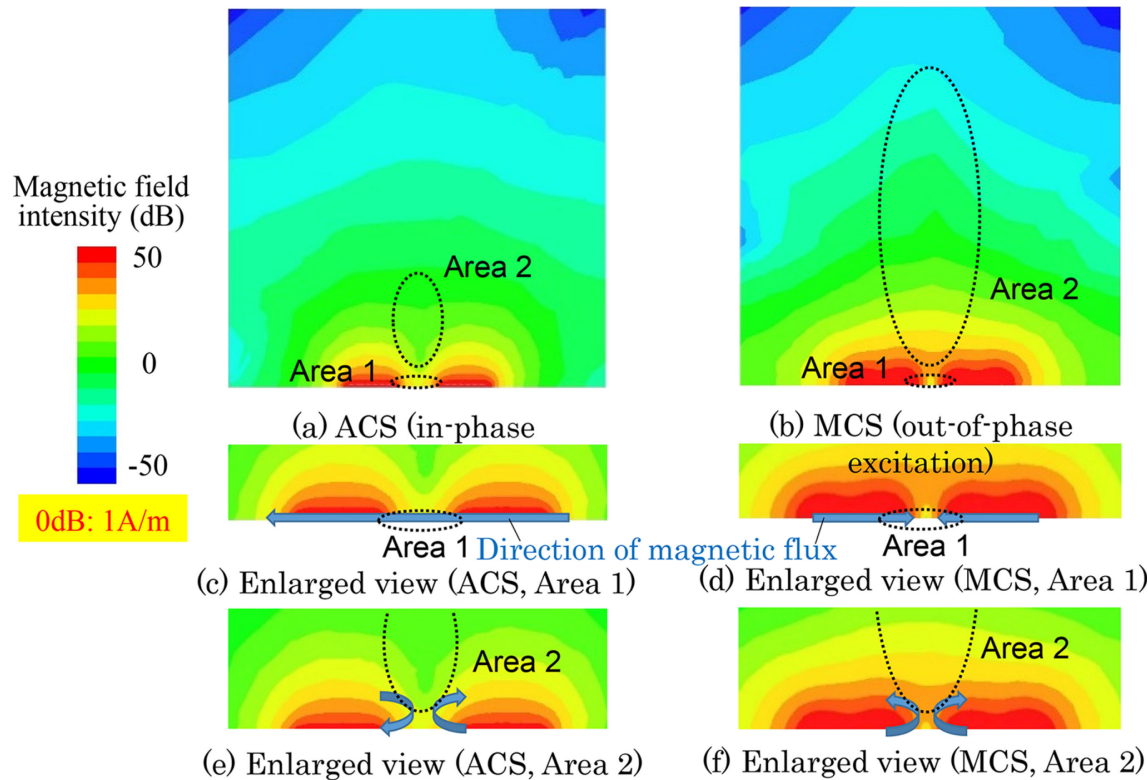


magnetic field of the in-phase excitation parallel to the solenoid is the same in the left and right solenoids. This is the reason based on which the magnetic field strength of the ACS array with the out-of-phase excitation rapidly decreases as $\mathrm{Z}$ increases, and the magnetic field strength of the in-phase excitation exceeds that of the out-of-phase excitation when $\mathrm{z}$ is larger than $20 \mathrm{~mm}$ (Fig. 2).

Although the MCS array yields results that exhibit similar tendencies, the magnetic field strength of the out-of-phase excitation is larger than that of the in-phase-excitation within the observed area $(\mathrm{z} \leqq 100 \mathrm{~mm})$. This is because the magnetic field produced in the solenoid is increased by the magnetic material so that Area 2 is enlarged. Therefore, it can be inferred that the excitation suitable for the NFC depends on the existence of the magnetic material.

\section{Conclusions}

This study investigated the influences of feed excitation in the case of the linearly aligned, two-solenoid array, on the magnetic field strength. As indicated by the results, the out-of-phase excitation can yield larger magnetic field strengths compared to the in-phase excitation near the center of the solenoid array because the magnetic fields produced by the two solenoids are added in this area. When the observation point is away from the solenoid, the in-phase excitation can yield larger magnetic field strengths than the out-of-phase excitation because a) the magnetic fields parallel to the solenoids are added and b) the magnetic field parallel to the solenoid becomes dominant. When a magnetic material is used to load the solenoid, the size of the loop of the magnetic field is enlarged because the magnetic field produced by the solenoid is increased owing to the magnetic material. Thus, the area where the magnetic fields are added near the center of the array is enlarged. Therefore, it is found that the excitation of the linearly aligned, two-solenoid array is suitable for NFC, and depends on the material constant of the magnetic material and the target distance between the solenoid array and the receiving antenna. In the future, we envisage conducting additional experiments and simulations to evaluate the transmission efficiency based on the consideration of the receiving antenna and the verification of the results with experimental measurements.

\section{Acknowledgments}

We thank Haruhiko Kondo of SMK Corporation for helpful discussions. 